\title{
3D Scanning For Hand Orthotic Applications: A Comparative Assessment Between Static And Real-Time Solutions
}

\author{
Paola VOLONGHI ${ }^{1}$, Alberto SIGNORONI ${ }^{* 1}$, Gabriele BARONIO ${ }^{2}$ \\ ${ }^{1}$ Università degli Studi di Brescia, Dip. di Ingegneria dell'Informazione, Brescia, Italy; \\ ${ }^{2}$ Università degli Studi di Brescia, Dip. di Ingegneria Meccanica e Industriale, Brescia, Italy
}

DOI: $10.15221 / 16.061 \quad$ http://dx.doi.org/10.15221/16.061

\begin{abstract}
The personalization of medical devices has made considerable progress in recent years. In the orthopedic field, following a typical reverse engineering approach, 3D scanning of anatomical regions of interest is the starting point for various kind of customized manufacturing, followed by the design of the device and its fabrication, particularly with additive manufacturing techniques. In this context, particularly challenging issues emerge from the customization of hand orthotic devices. The present work focuses on the identification and comparison of suitable scanning procedures in order to acquire accurate hand and fingers 3D models. Two different types of structured light scanners (static and real-time) were compared and several configuration of the hand were studied and acquired, allowing to evidence strength and weakness of the various approaches, while keeping in consideration the target application. In particular, the issues related to the presence of involuntary movements during acquisition are considered and possible solving approaches indicated.
\end{abstract}

Keywords: 3D hand scanning, structured-light scanners, real time scanners, orthotics.

\section{Introduction}

Manufacturing based on mass customization is expanding in medical applications. According to typical reverse engineering approach, customized devices can be obtained by digitization of the anatomy of interest followed by a modeling of the obtained surface and realization of the personalized device, typically using additive manufacturing.

Focusing on the upper limb, and in particular on the hand treatment, one of the most important target is the design and construction of orthotic devices, that can be useful for several pathologies and temporal or permanent disability conditions. For example, in case of hemiplegic patients, hand splinting is a very common treatment, even if there is much controversy surrounding real effects of rehabilitation using orthosis [1]. The clinical aims are the reduction in pain and spasticity, prevention of muscle and joint contracture and edema, improvement of function [1, 2]. In most cases, patients are compelled to choose fitting prefabricated orthosis among limited available sizes, which could be discomforting and painful [3]. Customization of orthotic devices becomes of great interest from a therapeutic point of view, since long-term compliance and tolerability are compulsory requirements.

Customization could be interesting also for other types of application, which are mentioned here even if they were not the main guiding factors of the present work. Anthropometric measuring, to build or complete a dataset that can be useful, for example, for gloves industry [4], is one of the possible final goal of a reverse engineering procedure. The availability of digital 3D anatomy is very interesting to avoid measurement directly on the hand of the subject with meters and goniometers, reducing discomfort. A further possible application is prosthetic design and fabrication. Anatomical information acquired with scanners can be useful to design a socket that is perfectly compliant with the anatomy of the patients, involving less contact pressure at the residual limb. Furthermore, scanners can be used to acquire the anatomy of the opposite healthy limb/hand/fingers, then a prosthesis can be designed and $3 \mathrm{D}$ printed, mirroring the reference geometry.

Nowadays, among the technologies available to acquire wrist/hand surface, it is worth to mention hand cast, photogrammetry and light-structured methodologies. The creation of a cast is the first and still the main currently used method, but it is the most invasive way to obtain personalized orthosis; it consists in manufacturing negative plaster cast, later filled with plaster to obtain the device, or in forming thermoplastic material directly on the hand of the patients. Due to its discomfort and painful characteristics, alternative non-contact methodologies have been developed in the years. An interesting device recently presented on the market specifically designed for hand scanning is Curatio (http://corporate.vectory3.com) which is based on photogrammetric technology and is able to take simultaneously multiple photos from various concentric angles distributed on a circumference to

*corresponding author e-mail: alberto.signoroni@unibs.it 
generate a 3D surface of the hand. The quick acquisition procedure avoids problems correlated with involuntary movements, but due to the way it is designed this technique presents difficulties in the acquisition of complex geometries with high accuracy (as fingers) and in avoiding occlusions caused by particular hand poses (especially the pathological ones requiring correction). This seems to limit its use only to simple orthoses (e.g. not including fingers). Another way to obtain 3D hand models in a non-invasive way and with the concurrent possibility to guarantee high accuracy and free pose scanning, is to use structured-light scanning technologies. Structured-light optical scanners can be "static" (this is not a standard name but it is used here to indicate scanners that works like a camera) or real-time (i.e. working like a video camera). Static scanners usually project a sequence of coded light patterns and are able to acquire single range scans in a time of the order of seconds (also depending on light and exposure conditions) and they require multiple acquisitions from different viewpoints to guarantee the complete coverage of the geometry of interest. In our case, involuntary hand movements are unavoidable factors that influence the acquisition (both intra- and inter-scan), constituting a problem that must be properly handled. Real-time scanners, instead, are based on fixed light patterns and they are portable and handy devices; the different views are continuously acquired at video frame rates and integrated (by fast close-range point sets alignments) while the scanner is moving slowly around the object. In comparison with static scanners, real time scanners imply a faster and easier acquisition of the 3D geometry but they are still partially influenced by patient movement. The present work is focused on the identification of suitable scanning procedures and the analysis of residual problems in a perspective of hand modeling for orthotic applications. Two different scanner technologies were compared: static multiview and real-time incremental acquisitions. We used two products from Open Technologies Srl, Rezzato (Brescia), Italy: the static 3D scanner Cronos 3D Dual and the real-time scanner Insight3. For each technique, potentialities and main issues, with possible solutions, are analyzed, in order to define a proper acquisition strategy depending on the anatomical portion of interest and related application.

\section{Methods}

The production of personalized orthosis can follow a typical reverse engineering / rapid prototyping process, that can be divided in three main phases: a) Acquisition of the anatomy of interest of the subject (Fig.1A); b) Processing of the acquired geometry using suitable software (Fig. 1B); c) Printing of the orthosis through 3D printer (Fig. 1C). The second phase is critical and a review and discussion of main issues can be found in Baronio et al. [6]. For the third phase, despite additive manufacturing is always interested by relevant innovation trends, quite consolidate 3D printing technologies already exist and they can be chosen in respect to specific needs. For these reasons, in this work we focus on the first phase which, in the case of the typical issues related to hand acquisition, has not yet been sufficiently addressed in literature. We start from the evidence of the possibility to acquire the 3D geometry of the hand using static 3D scanner [6], extending the analysis to the comparison of different technologies.
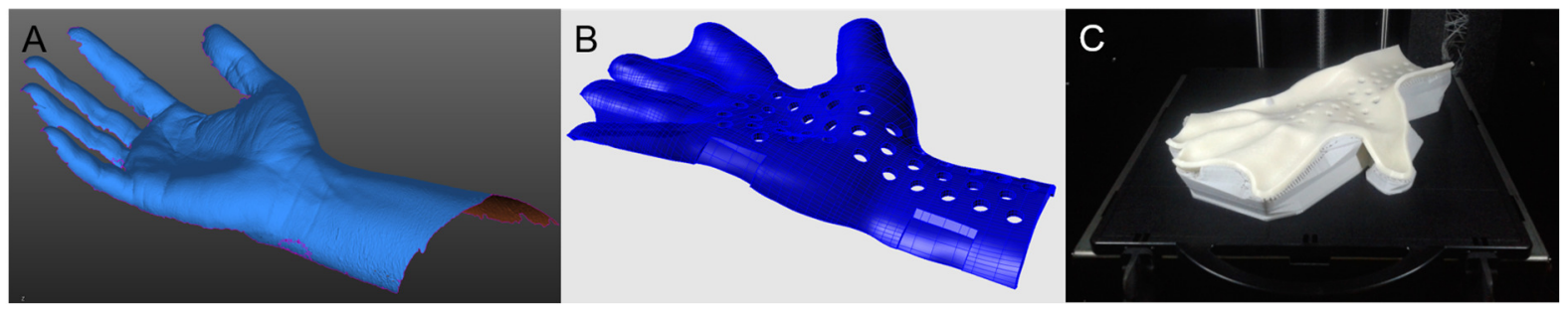

Fig. 1. Reverse engineering / Rapid prototyping process phases.

\subsection{Scanner technologies and acquisition modalities}

For the present work, two different types of scanner were used: Cronos 3D Dual (figure 2, left) and Insight3 (figure 2, right), both produced by Open Technologies Srl (http://www.scanner3d.it/en/).

Cronos is a professional structured-light static scanner capable to acquire range scans of surfaces with high accuracy (about $\pm 30-60 \mu \mathrm{m}$ depending on the working field setup). It is mounted on a tripod and can be used in two different ways: a) scanner fixed on its tripod and the object placed on a rotating table or freely moved in front of the scanner, b) scanner on the tripod moved conveniently around the steady object. Obviously, the first modality is the more practical one, but the second is the only one we found workable for hand scanning which is intrinsically susceptible to involuntary movements. As an 
advantage, once the hand is suitably positioned and the subject can place the limb on supports that help to keep the hand steady, the acquisition of the various 3D views is quite unconstrained and the operator can select the best vantage points to avoid occlusions in the final model. However involuntary movements can affect the acquisition in two different ways: a) intra-scan movements are referred to the subject moving the hand/fingers during the short time interval dedicated to a single acquisition; in this case, reconstruction artifacts can appear in the acquired range image and there could be the need to repeat the single scan; b) inter-scan movements are difficult to avoid and seemingly disqualify the use of a multiview acquisition for non-rigid objects; however if the total acquisition time is not causing discomfort to the subject in keeping the hand in the desired position, small movements occurring among scans can be compensated. The scanner software (Optical RevEng by OpenTechnologies) allows a so called direct multiview alignment which is a markerless geometric alignment of the different views. We verified that this modality is capable to automatically produce a pretty well aligned set of scans even in presence of small to moderate hand and finger motion (each new acquired scan from a freely chosen vantage point is automatically and instantaneously aligned to the previous set). This is an ideal starting point for a subsequent deformable alignment for the compensation of inter-scan movements. This was easily obtained by a technique developed by our group [5], based on patch-wise as-rigid-as-possible deformable adjustment of partially overlapping scans.

Insight3 is a real-time scanner operating with a fixed structured-light technology (in the infrared range) and it works like a video camera. It does not require a tripod, it can be easily operated thanks to its lightness and to the possibility to grasp it with two hands. Its nominal accuracy is about $\pm 0.25-0.5 \mathrm{~mm}$, hence one order of magnitude worse than that of Cronos. The scanner software allows views accumulation at video frame-rates over a large working area, the markerless (a very relevant feature for medical applications) frame tracking mechanism requires the operator to move the scanner smoothly around the object of interest. Each scan acquired under valid tracking is integrated in the model and contributes to the scanning noise reduction that, for single frames, is quite visible (a known limit of fixed light pattern acquisitions). The software gives real-time visual feedback about the tracking state and the quality of the reconstruction which are very informative and allow the operator to properly guide the scanning and to recover the tracking state if lost during the scanning. Even if the scanner has to be moved slowly, the acquisition is faster compared to static scanner, but not enough to avoid problem due to involuntary movements. Small tremors are mediated, but true displacements are integrated, possibly producing unwanted artifacts on the final model. For this reason, it is very important that the subject, assisted by a faster acquisition, avoid every type of movement during the scanning.

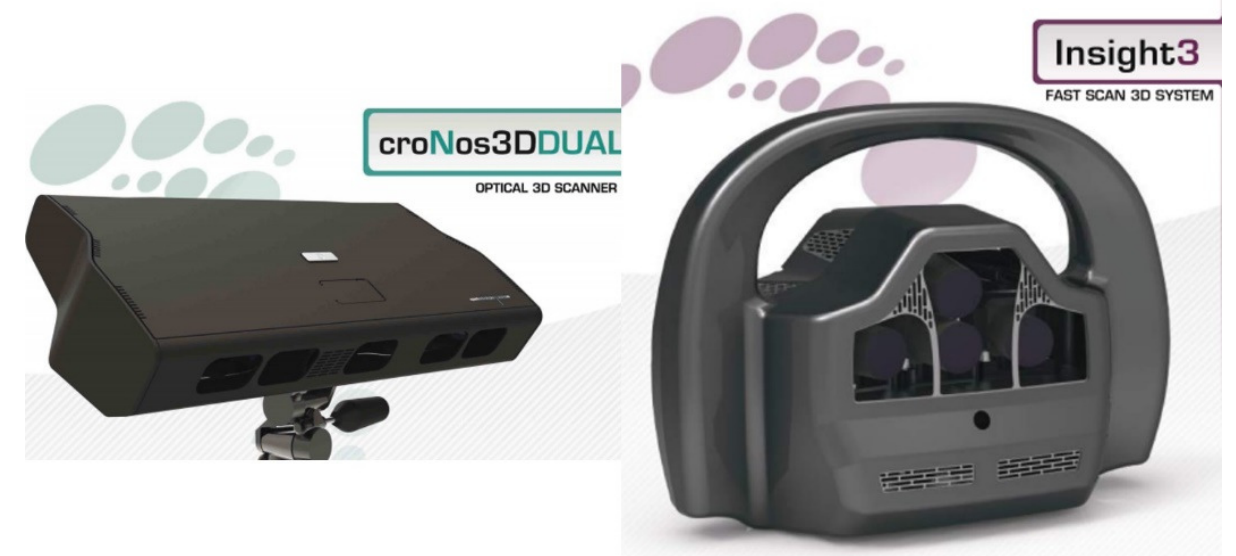

Fig. 2. Static scanner (Cronos) and real-time scanner (Insight) illustrations.

\subsection{Post-processing}

The software Optical RevEng v.2.4 controls both scanners and operates both the measuring and data processing processes. The aforementioned automated markerless direct alignment is probably the most important available tool for static scanner acquisitions. Aligned range images are saved and loaded from the separate deformable alignment software where every scan is aligned to the one chosen as a reference. In accordance with what described in [5], our deformable alignment is capable to handle small to moderate displacements, therefore covering the whole spectrum of involuntary movements one may sensitively want to compensate. The so aligned scans are reloaded to the proprietary software that can handle further data cleaning, mesh model creation and editing. For example, a series of powerful (semi)automated mesh fixing and denoising tools are available to finalize 
the anatomical model. In this work, however, we focus on the first phases of acquisition by assessing their workability in a perspective they could be taken as reference for real applications. Therefore, only essential data handling is made here, also to not disguise the acquired models that we want to show as close as possible to the original data.

\subsection{Hand modeling targets}

According to considerations and needs emerging from an analysis of what anatomic acquisition should represent for orthoses design, 4 different hand modeling target can be indicated. They are 1) palmar acquisition, 2) dorsal acquisition, 3) whole hand, 4) customized partial acquisitions. The most relevant targets for orthotic applications are 1) and 2), while 3) is more interesting for prosthetics and 4) for specific devices or aiding tools.

In order to define optimized acquisition procedures depending on the scanner technology and target applications, several tests were devised. The aspects of major interest are ease of use, total acquisition time, completeness and accuracy of the final obtained geometry.

Preliminary tests were made on a mannequin hand to study some feasibility and quantitative aspects of the acquisitions. In this case the scanner was kept fixed and the mannequin hand was arranged vertically on a rotating table. For the static scanner, a redundant set of 24 views were scanned, with a regular step angle of $15^{\circ}$, in order to identify a-posteriori (by view removal) the minimum number of acquisitions needed to obtain a complete coverage of hands in normal pose.

Other tests involved the scanning of volunteers' hands and the use of both scanners. Volunteers were asked to position their hand on a pillow (placed on a table) and to maintain as steady as possible, avoiding coarse movements, while the scanners were moved around. The number of views was adjusted for static scanner (8 evenly spaced views were judged enough for both palmar and dorsal models) and different modalities of scanning were tested for real time scanner, in order to establish acquisition guidelines. Palmar and dorsal sides were acquired using both scanners, while the entire hand geometry was acquired only with the real-time scanner. In this preliminary study, whole hand acquisition with static scanner was excluded mainly for the longer time required (few minutes) and the consequent difficulty to maintain the position, especially of the fingers. Moreover other issues arose while using a single-reference deformable alignment of the various scans when both palmar and dorsal views are present.

\section{Results and Discussion}

In this section, results and discussion are reported, subdividing the paragraph in different parts, each one analyzing the several tests performed. The models presented here underwent only essential post-processing of the acquired geometries. This consisted in removing background objects (as the table), performing direct alignment (for static scanner), executing deformable alignment (for static scanner, when required) and improving the reconstructed mesh, with essential mesh fixing (small holes filling and a reduction of the number of triangle by decimation).

\subsection{Preliminary tests}

The time required for the acquisition of the 24 views of the mannequin hand in the vertical position was 2' 40 ". Since, due to the semi-closed hand pose, the nails surface was not captured by the common vantage point, 12 additional views of fingertips were acquired (with prone hand) rotating the platform only by $180^{\circ}$, with a step angle of $15^{\circ}$, for an acquisition time of $1^{\prime} 30^{\prime \prime}$. Direct alignment of the two set of views allowed to obtaining, in a few seconds, an almost complete mesh (figure $3 \mathrm{~A}$ ). Few micrometric double skin imperfections on a small area were present, probably due to localized light reflections, which can be easily corrected with mesh fixing tools. We noticed that dividing in a half the views (12 for vertical position, 6 for the prone one), the mesh did not show imperfections anymore. The number and dimension of holes increased (figure 3B), but they were successfully filled applying the automatic tool supplied by the scanner software (figure $3 \mathrm{C}$ ). A further reduction of the views was performed: considering a step of $45^{\circ}$ for prone and vertical position (reducing the views from 36 to 14 and the time acquisition from about 4 minutes to less the 2 minutes), an acceptable mesh was again obtained, without surface imperfections and with holes reparable with the available automated hole filling tool (a picture is not reported because it is very similar to figures $3 \mathrm{~B}$ and $3 \mathrm{C}$ ). Therefore, a step of $45^{\circ}$ is to be considered sufficient and also suggested for the acquisition of the entire 3D surface of a mannequin hand with a static scanner. 


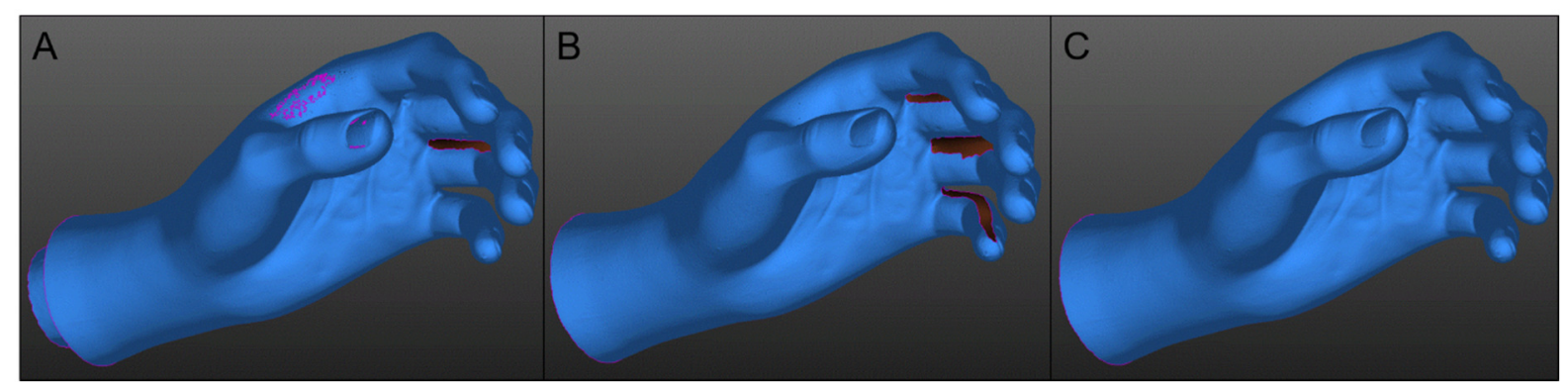

Fig. 3. Mesh surfaces of the mannequin hand obtained with static scanner. A) Resulting mesh from 36 views (24 vertical and 12 prone), imperfections and holes are showed; B) Resulting mesh from 18 views (12 vertical and 6 prone), holes are showed; C) B mesh corrected with hole filling tool.

\subsection{Volunteers hand acquisitions}

Considering the real hand scanning, both scanners were used to reconstruct dorsal and palmar side. We primarily considered the geometry of only one side of the hand because, in addition to be easier, it is also sufficient and recommended for orthoses (and also for anthropometric applications). Later, we dealt with total hand scanning.

\subsubsection{Static scanner - one side hand}

Using static scanner, dorsal side of the hand was acquired in less than 3' (8 views) and the resulting mesh is illustrated in figure 4 (top raw). A great part of the acquisition time is due to the time required to manually move the tripod in the different positions. Post-processing steps only implied removal of the table and a piece of the forearm, and the direct alignment of the views. The obtained mesh showed little imperfections, that appeared as double skin (figure 4A), probably due to slight involuntary movements, and they were corrected applying the deformable alignment algorithm, as it can be visible in figure 4B. We noticed that reducing the views from 8 to 4, the quality of the mesh didn't worsen, on the contrary it didn't present double skin, probably due to the less superimposed information; the only detriment is the loss of information at the boundary of the hand, where the surface changes its curvature.

Also palmar side acquisition with static scanner required about 3' (figure 4, bottom raw). Considering all 8 views, the double skins were more evident than that obtained in dorsal side (figure 4C). This is due to a higher difficulty in maintaining the hand steady in a supine position with respect to the prone one. However, these issues were again easily corrected by the application of the deformable alignment technique (figure 4D). Taking in consideration only 4 views (1'20" acquisition time) instead of 8 , the loss of information was admissible, while small movements imperfections were anyway present; then the application of the deformable alignment technique was anyway necessary.

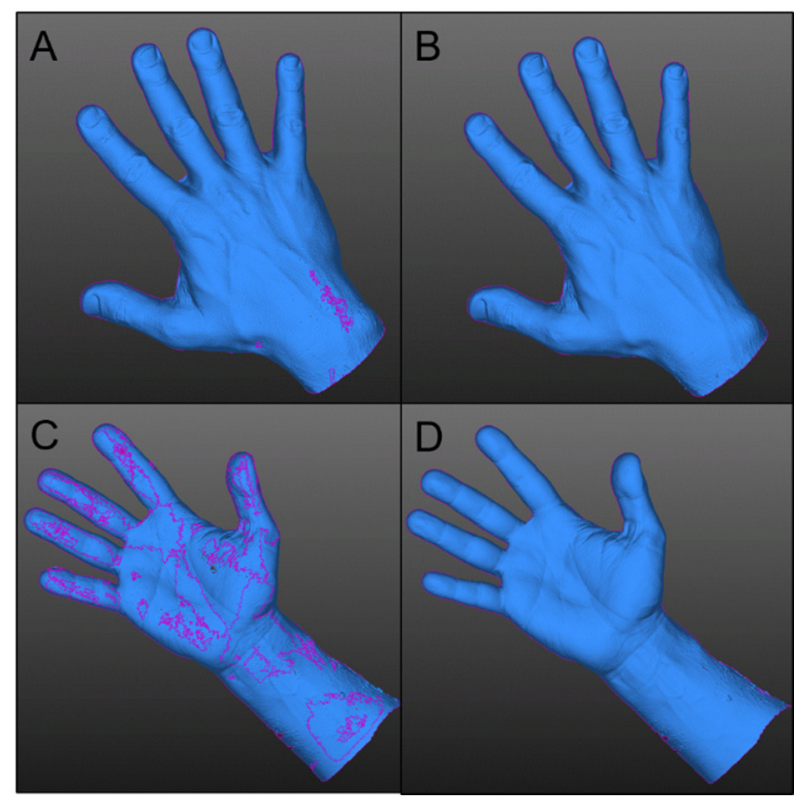

Fig. 4. Resulting mesh of static scanner acquisitions. Dorsal side is showed in the upper raw, while palmar side in the lower. Original meshes $(A$ and $C$ ) were corrected applying the deformable alignment algorithm $(B$ and $D)$. 
We can conclude that, in case of static scanner, 4 evenly spaced views are sufficient to build a complete and acceptable one-side geometry of the hand in a normal pose, allowing reliable personalized orthosis construction or anthropometric measures.

\subsubsection{Real time scanner - one side hand}

Real time scanner allowed a reduction of the acquisition time. Considering orthotic application, we suggest to move the scanner around the hand maintaining a side view, starting from the little finger side; the acquisition time was about 1 minute. Remember that this is a completely noninvasive and markerless procedure, the absence of markers imposed a not too fast scanner displacement in order to allow the scanner tracking system to work properly. The hand was in a neutral position for palmar side (figure 5A), while it grasped a little sphere (chosen to help the volunteers to maintain a neutral position) for dorsal side (figure 5B).

For anthropometric applications, simplified acquisition can be performed, since an accurate boundary surface of the hand is not necessary. In these cases, we suggest to move the scanner following a top view of the hand, starting from the forearm and finishing to fingertips. For both palmar and dorsal side (an example is given in figure $5 \mathrm{C}$ and $5 \mathrm{D}$ ), the hand was positioned flat on the table. In this case the acquisition time was further reduced to less than 20 ".

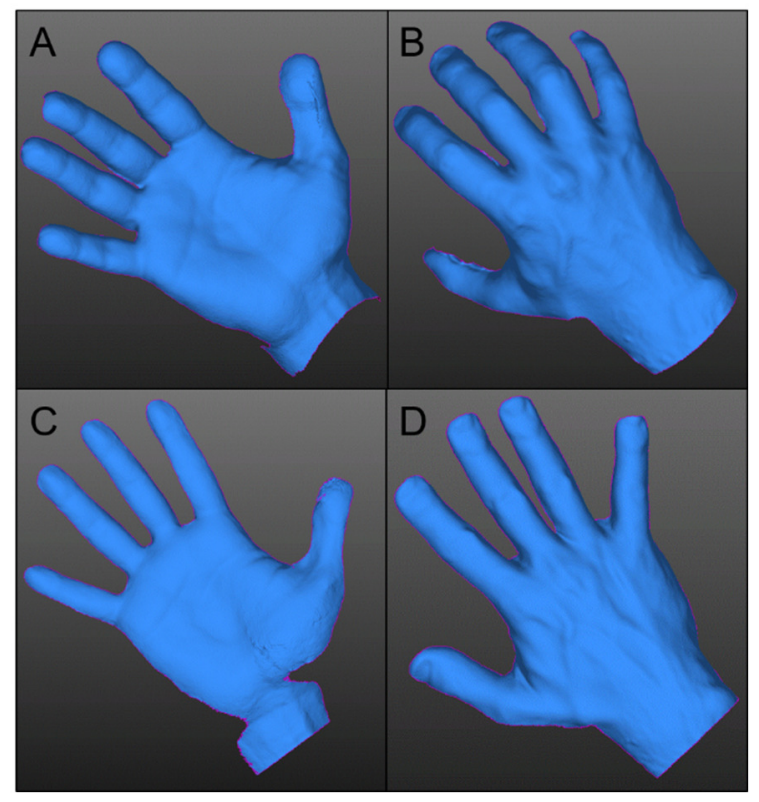

Fig. 5. Resulting mesh of real time scanner acquisitions. More complete geometry is shown in $A$ and $B$, than more simplified model is illustrated in $C$ and $D$.

\subsubsection{Level of detail}

Level of detail reached by real-time scanner is good, but it is far from what we can obtain with static scanner (see figure 6 for a comparison). Notably, for static acquisitions, we verified that the detail level is not influenced by the application of the deformable alignment algorithm. On the other side, for real-time scanned surfaces, low entity motion artifacts (as the one that can be noticed in figure 6B) can be easily repaired. Despite evident, the diversity in terms of surface detail reached by the two scanning technologies is not expected to be a discriminant for the realization of customized orthoses; in fact, this requires good metric accuracy but not at the level of high-fidelity skin texture reproduction.

\subsubsection{Total hand scanning}

The main difficulties were encountered for total hand acquisitions. Volume geometry is required for prosthetic application, less for orthotic (in fact, orthosis on the market are only one side devices). It is anyway interesting to analyze this case, in order to assess the feasibility of the scanning. As stated, we discarded the use of the static scanner. We asked volunteers to keep the hand in a neutral position, as comfortable as possible. At first instance, acquisitions resulted problematic, the real time scanner struggled to maintain object tracking, with difficulties to accumulate views automatically. 


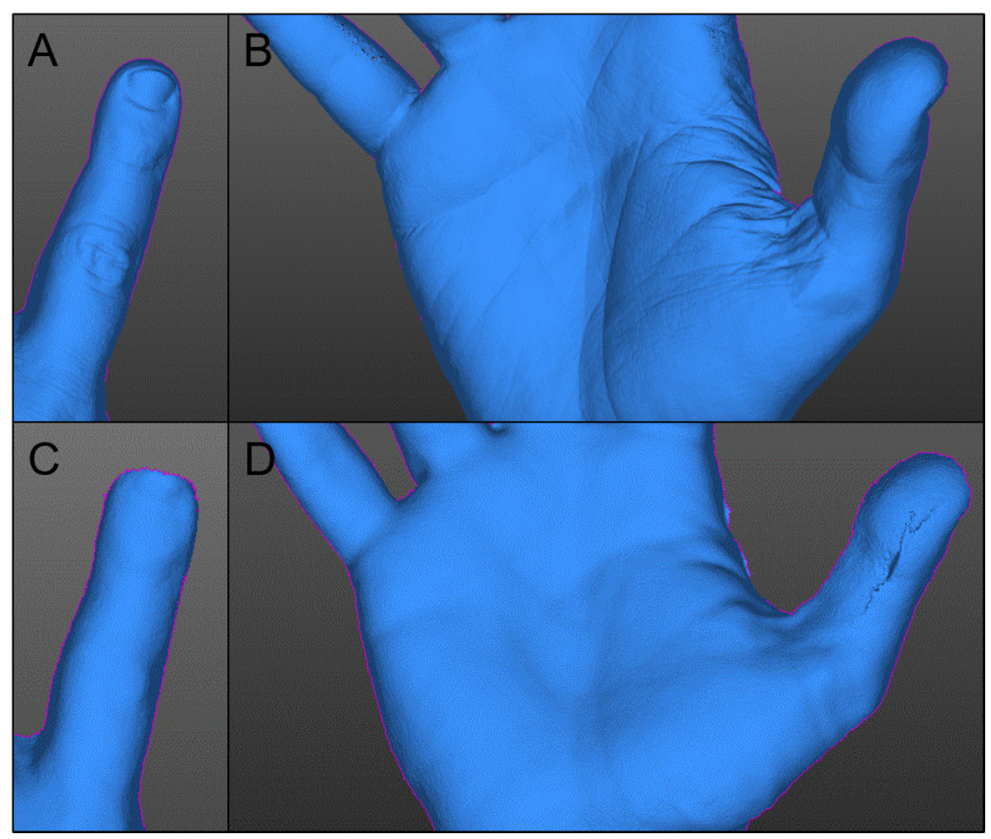

Fig. 6. Diversity of details reached using static scanner (top raw) and real-time scanner (bottom raw). $A$ and $C$ pictures show little finger detail in a dorsal view, while $B$ and $D$ figures represent palmar details.

This was not so much due to the specific scanner, but on the fact that the passage from one hand side to the other occurs while the hand appears very thin to the scanner, so that the tracking problem quickly become ill-conditioned (the acquired surface is very reduced and may easily become floating, with the consequence that it cannot be univocally integrated into the accumulating surface). This produced unsatisfactory results or objectionable "loop closure" artifacts. To work around the problem, we suggest to use additional devices to prevent this "thinning" effect and allow the real time scanner to keep the alignment in order to obtain the complete $360^{\circ}$ acquisition. For example, we realized a very simple support consisting in a double wire coiled around two fingers of the hand, with two tennis table balls at the extremities (figure 7A). Figure 7B depicts the usage of this tool during scanning. This was an expedient to increase the surface the scanner sees while turning around the hand from one side to another. Many other, even less invasive, ways to fasten some targets to the hand or to the wrist can be devised to appear in the field of view to maximize the framed surface when the visible hand's surface is reduced, but the design of such devices is out of the scope of our study. What we noticed is that the spherical shape helped the scanner to maintain the tracking state active and allowed a successful whole hand scanning, where about 3 minutes were required for acquisition. Figure 8 depicts the resulting surface prior to any improving post-processing (only ring and sphere removal with due hole filling was accomplished). Residual artifacts were due to hand motion during the scanning time. Despite preliminary, these outcomes can be taken as a guide for future works where, for similar cases, either marker based alignments or the development of more effective supports can be considered, in order to simplify the acquisition, reduce its duration and help the subject to keep a stable pose.

\section{Conclusion}

The principal aim of this work was to identify suitable hand acquisition strategies using different kind of structured light scanners, as an alternative to cast and photogrammetric techniques. Use of cast to acquire the geometry of the patient is extremely invasive and discomforting. The use of photogrammetric solutions has the significant advantage of very fast acquisitions (in less than a second a several images can be acquired simultaneously), but the disadvantage of the inability to capture areas that are occluded due to particular positions of the hand and fingers (indeed, the hand is rarely acquired totally open for orthotic applications).

A comparison between static and real time scanners has been performed. Static scanner is commonly suggested for inanimate objects and when high accuracy of the geometry is required. We verified that involuntary inter-scan movements can be perfectly compensated by an advanced deformable alignment solution. In case of hand acquisition for orthotic and anthropometric applications, in which only one side geometry is required, high accuracy (as $\mu \mathrm{m}$ provided by Cronos) could not be considered a compulsory plus. Thanks to its hand-held nature and markerless tracking, the considered real time scanner allows faster and easier acquisitions, while providing good enough accuracy. 


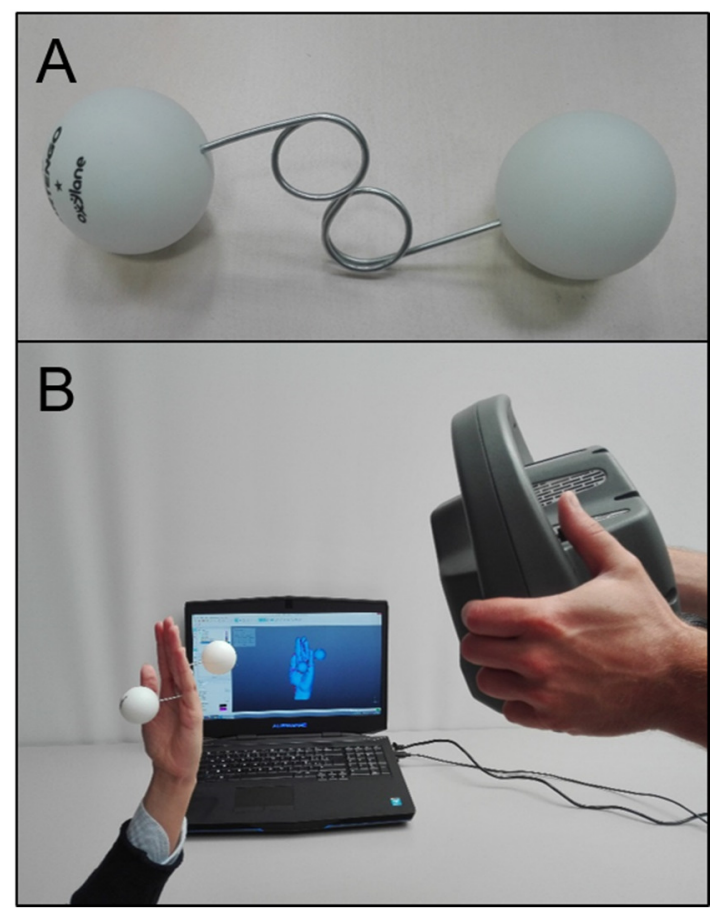

Fig. 7. A) Realized support. B) Typical scanning setup.

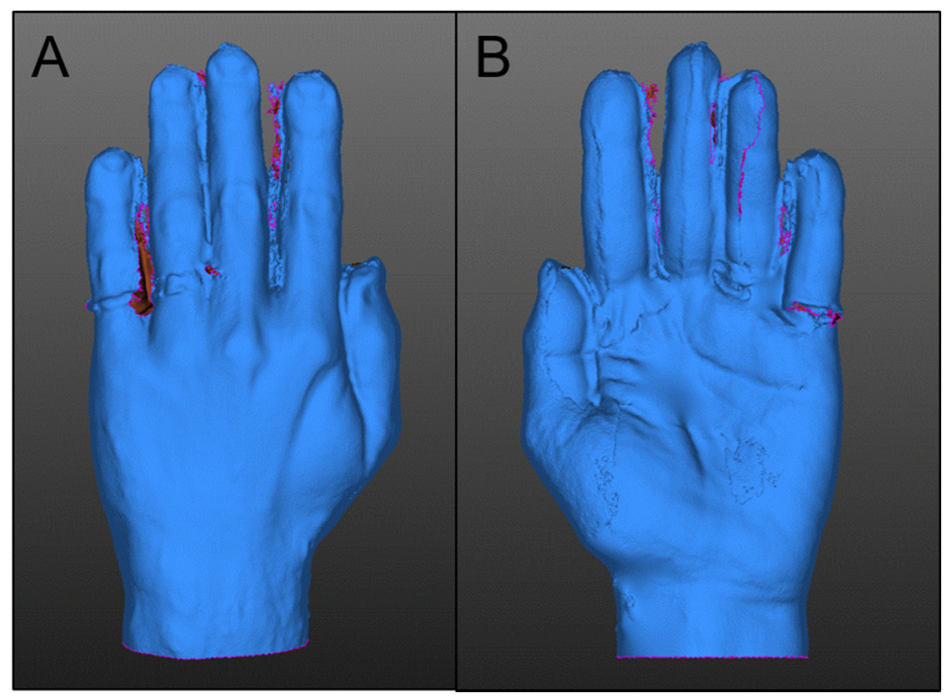

Fig. 8. Dorsal (B) and palmar (C) side of the hand acquired with static scanner.

This study demonstrated that hand modeling for orthotic applications, i.e. requiring single sided palmar or dorsal hand scanning, is totally feasible with both static and real-time scanner technologies. Differently, total hand acquisitions evidenced some open issues that were only partially solved in this work.

A wider trial be started including patients and involving, where necessary, the design of comfortable supports or grip systems to facilitate the maintenance of the position of the hand during the scanning.

\section{Acknowledgments}

This work is a preliminary study included in a wider project (RESHAPER, Reverse Engineering of Self-care and Healthcare Aids for Personalized Empowerment and Rehabilitation), funded by the University of Brescia and Open Technologies Srl, in cooperation with Fondazione Teresa Camplani Casa di Cura Domus Salutis and Partec Srl. 


\section{References}

[1] N. A. Lannin and R. D. Herbert, "Is hand splinting effective for adults following stroke? A systematic review and methodologic critique of published research," Clin. Rehabil., vol. 17, pp. 807-816, Dec, 2003.

[2] E. Bürge, D. Kupper, A. Finckh, S. Ryerson, A. Schnider and B. Leemann, "Neutral functional realignment orthosis prevents hand pain in patients with subacute stroke: a randomized trial," Arch. Phys. Med. Rehabil., vol. 89, pp. 1857-1862, 2008.

[3] A. Andringa, I. van de Port and J. W. Meijer, "Long-term use of a static hand-wrist orthosis in chronic stroke patients: a pilot study," Stroke Res. Treat., vol. 2013, pp. 546093, 2013.

[4] A. Klepser, M. Babin, C. Loercher, E. Kirchdoerfer, J. Beringer and A. Schmidt, "3D Hand Measuring with a Mobile Scanning System," Proc. of 3rd Int. Conf. on 3D Body Scanning Technologies, pp. 288-294, 2012, http://dx.doi.org/10.15221/12.288.

[5] F. Bonarrigo, A. Signoroni and M. Botsch, "Deformable registration using patch-wise shape matching," Graphical Models, vol. 76, pp. 554-565, 2014.

[6] G. Baronio, S. Harran and A. Signoroni, "A Critical Analysis of a Hand Orthosis Reverse Engineering and 3D Printing Process," Applied Bionics and Biomechanics, vol. 2016, 2016. 